\title{
Recurrence Plots of Geolocated Time Series from Satellite Maps of NOAA STAR Vegetation Health Index
}

\author{
A. C. Sparavigna ${ }^{1}$, R. Marazzato²
}

\author{
${ }^{1}$ Department of Applied Science and Technology, Politecnico di Torino, Torino, Italy \\ ${ }^{2}$ Visiting Staff at Department of Mathematical Sciences, Politecnico di Torino, Torino, Italy
}

\begin{abstract}
Several information services, such as the NOAA Center for Satellite Applications and Research, produces and distributes maps elaborated from satellite images, which display data about vegetation indices. Using the timeseries concerning some specific geographical positions, which we can obtain from the available maps, several analyses are possible. Here we propose the use of recurrence plots. We will show examples based on the data corresponding to six small areas, geolocated in Italy, of the NOAA STAR Vegetation Health Index (VHI). It is an index used for monitoring and forecasting the status of vegetation. The recurrence plots we obtained could be helpful for discriminating different situations.
\end{abstract}

Keywords: Satellite Maps, Satellite Imagery, Recurrence Plots, Vegetation Indices, Climate

\section{Introduction}

In 1987, Eckmann, Oliffson Kamphorst and Ruelle introduced the recurrence plots, a simple way of visualizing the periodic or chaotic behaviour of a dynamical system through its phase space [1]. The researchers presented these plots as a diagnostic tool to test the evolution of a dynamical system, to be used besides the parameters already involved in the analysis of time series, such as information dimension, entropy, Lyapunov exponents and spectra. Soon after their introduction, the recurrence plots have been used in investigating natural processes, which can have quite distinct recurrent behaviours; using them, we can observe short term seasonal periodicities or long term natural cycles, but we can also display the irregular behaviour of El Niño Southern Oscillation [2,3]. In this framework of environmental analyses, we have shown, for instance, the seasonal oscillations of levels of some lakes in Africa [4], and the trend of carbon dioxide concentration in atmosphere, linking it to $\mathrm{CO}_{2}$ emissions of anthropic origin [5].

In environmental studies, several data are given as time-series of specific quantities, obtained from different sensors. When global data are involved, averages of several observations in different locations are often used. Among devices that collect environmental data, we have the satellites equipped with hyperspectral sensors, specifically made to detect electromagnetic radiations at different wavelengths. In this manner, besides time-series of satellite images in the visible range, which can be used for investigating the evolution of local landscapes (see for instance, in [6,7], the monitoring of motion of sand dunes), we have several imageries in other frequency ranges which contain data from remote sensing of temperature, moisture, water use and other environmental parameters. Each pixel of the image covers a certain geographic area. Therefore, we can consider each pixel of these images (or an average of some pixels) as a geolocated environmental sensor and we can use the corresponding time-series of data for monitoring and forecasting. In fact, we can also use them to create recurrence plots of vegetation indices [8]. Besides recurrence quantification analysis [9], the recurrence plots can be a useful tool for identifying specific patterns in environmental phenomena concerning the vegetation indices [10].

Here, we will show and discuss some examples based on the NOAA STAR Vegetation Health Index (VHI), an index that was proposed for monitoring the status of vegetation. NOAA STAR is the Center for Satellite Applications and Research of the National Oceanic and Atmospheric Administration (NOAA), a federal agency of United States, focused on the

This article is published under the terms of the Creative Commons Attribution License 4.0

Author(s) retain the copyright of this article. Publication rights with Alkhaer Publications.

Published at: http://www.ijsciences.com/pub/issue/2015-12/

DOI: $10.18483 /$ ijSci.877; Online ISSN: 2305-3925; Print ISSN: 2410-4477 
condition of the oceans and the atmosphere. The examples discussed in this paper refer to six small areas in Italy. We will see that recurrence plots seem able of discriminating different situations. We will also show that these plots are quite different from the corresponding plots that we can obtain from the maps of another largely used vegetation index, the NDVI, i.e. the Normalized Difference Vegetation Index.

\section{Vegetation monitoring}

Before showing and discussing recurrence plots from NOAA STAR data, let us shortly revise the satellite method for monitoring vegetation. The green plants absorb that part of solar radiation which lies in the photosynthetically active spectral range. Plants use it as a source of energy in the process of photosynthesis; moreover, they evolved to have leaf cells scattering solar radiation in the near-infrared spectral region. Hence, live green plants appear relatively dark in the photosynthetically active spectral range and relatively bright in near-infrared [10]. Therefore, leaves strongly absorb visible light (from 0.4 to $0.7 \mu \mathrm{m}$ ) for use in photosynthesis, whereas their cell structure strongly reflects nearinfrared light (from 0.7 to $1.1 \mu \mathrm{m}$ ). The more leaves a plant has, the more these wavelengths of light are affected. Since satellites, such as NOAA AVHRR, acquire data in visible and near-infrared, it is quite easy to observe the strong differences in plant reflectance to determine their spatial distribution and prepare some specific maps.

Based on satellite measurements, some vegetation indices can be given; one which is largely used is NDVI (Normalized Difference Vegetation Index), easily defined as follows: NDVI = (NIR-VIS)/(NIR+VIS), where VIS and NIR stand for spectral reflectance acquired in the visible (red) and near-infrared regions, respectively. The remotely sensed spectral vegetation indices are involved in numerous disciplines interested in the assessment of biomass, water use, plant stress and health, and crop production [11]. However, although vegetation indices were developed to extract the plant signal only, the soil background, moisture condition, solar zenith angle, view angle, as well as the atmosphere, alter the index values in complex ways [11].

The time series of NDVI or other indices can be used to obtain ecological indicators to forecast the ecology of a given region. In fact, as observed in [12], being a crucial component of terrestrial ecosystems, the vegetation dynamic has become one of the key issues in the study of global environmental change, so that many researches have been conducted on vegetation dynamic trend on different periods. In [12], the consistency of these trends after the study period was analysed using Hurst Exponent and R/S analysis. Hurst and Lyapunov exponents have been used also in [13-15]. More generally, Hurst exponent can be involved in fractal analysis of LANDSAT images [16], to investigate the local changes in land uses.

\section{NOAA STAR VH Index}

For what concerns the vegetation indices and related maps, several satellite imagery resources are available for investigations. NOAA STAR, a centre for satellite applications and research, provides many of them. This centre is the science arm of NOAA Satellite and Information Service (NESDIS), which acquires and manages Earth-observing satellites. NOAA STAR has the mission to accelerate the transfer of satellite observations from scientific research and development into routine operations. Among the several data provided by the site, we find those concerning the vegetation health, moisture and thermal conditions. Of the Vegetation Health (VH), the satellite observations are principally given by the Advance Very High Resolution Radiometer (AVHRR), flown on NOAA polar-orbiting satellites. Data are global with the resolution $4 \mathrm{~km}$ and 7-day composite. The center provides the following vegetation indices: Vegetation Condition index (VCI), Temperature Condition index (TCI), Vegetation Health index (VHI), Soil Saturation index (SSI), No noise Normalized Difference Vegetation Index (SMN), No noise Brightness Temperature (SMT), Fire risk index (FRI), and other products on drought and malaria.

The Vegetation Health Index (VHI) is based on a combination of VCI and TCI. It is considered effective enough to be used as proxy data for monitoring vegetation health and drought as discussed in [17]. VHI has been used successfully for detection of vegetation stress and estimation of crop losses in the USA. The data showed that large-area drought can be detected up to two months in advance if compared to other techniques, and the impact on grain reduction can be diagnosed long before in comparison to harvest [17]. Due to the importance of this index, we decided to use it for our analysis with recurrence plots, instead of the NDVI index used in [8].

\section{NOAA STAR data of Italy}

Among the data provided by NOAA STAR, we choose those concerning Italy. In the Figure 1 we can see two VHI maps: note that these maps are not the original satellite images but matrices of processed data. Here, we investigate the behaviour of small regions of a few pixels, corresponding approximately to 30-square $\mathrm{km}$. First of all, we choose three areas. These are in the northern part of Sardinia (Santa Teresa di Gallura, Rena Majore, Li Lieri and Pulcheddu), the area of Trieste and the wedge of land lying between rivers Po and Ticino near Pavia (San Martino Saccomario, Carbonara al Ticino and Cava 
Manara). The aim is that of understanding how local environment can influence the behaviour of VHI and, therefore, its forecasting capability at local scale. In the case of the above mentioned three areas, the environment is quite different: one is an insular environment in the middle of Mediterranean Sea (Sardinia), one is between sea and mountains (Trieste) and the last is in the Pianura Padana (Pavia). After this, we will consider three areas from the same region in the central Italy (Umbria), corresponding to Perugia, Norcia and Terni, to investigate the recurrence plots in the case of three locations which should have approximately the same environmental conditions.

NOAA STAR maps from the first week of 2010 to the last available of 2015 have been used. Data extraction has been made by means of a software, called SIDaX, which has been specifically developed for this purpose. Its interface appears in Figure 2: it allows the basic choices of images folder, data type, geographical points set, and name of the whole dataset. The result is a simple highly compatible CSV file containing the list of estimated index values for the selected points and for their near surroundings.

According to the scale in Figure 1, the highest numerical value of the index is given to the corresponding color tone. An average value on the chosen area is calculated and, as a consequence, a time series is obtained. The results corresponding to Sardinia, Trieste and Pavia are given in the graph of Figure 3. High values correspond to healthy vegetation, whereas lower values correspond to a stressed situation. In the following Figure 4, we can find the corresponding recurrence plots. To prepare them, we used E. Kononov software, in the Grey and Spectrum layouts [18]. In the Grey layout plots, black or dark grey pixels correspond to high recurrence, whereas white pixels to low or very low recurrence. A recurrence is a case the time-series datum returns to a value it has attained before. The plot is subdivided into macro areas separated by boundaries. In fact, the plots given in the Figure 4 seem to show a mixture of oscillatory and autoregressive processes (see for instance the examples of these two cases at [19]).

Of course, it is natural that oscillations are present, due to season effects. However, it seems more relevant the presence of an autoregressive process. In statistics, an autoregressive model is a representation of a type of random process where the output variable depends linearly on its own previous values and on a stochastic term (see [20] and references therein). In an auto-regressive process, a shock affects the evolving variable far into the future. In the case of vegetation, a shock can be represented by anthropic actions too. Shock effects are expected to be quite relevant on small areas. Let us note that, in the case of the area near Pavia, we have a strong stress of vegetation, which is connected to that observed for the Pianura Padana as a whole.

From Figure 4, we can see that, in the upper Grey layout recurrence plots, white areas exist which seem separating the image in chessboard textures; these areas are corresponding to the peaks in graphs. In the same Figure 4, coloured recurrence plots are also shown for easy comparison. Plots are obtained by means of Kononov software in Spectrum layout; using such a layout, the boundary regions between macro areas, which are evidencing peaks in timeseries, are clearly visible in green. Let us note that the recurrence plots show that the coastal areas of Northern Sardinia and Trieste have a similar behaviour, but the wedge Po-Ticino near Pavia is quite different and typical of the Pianura Padana environment.

In the Figure 5 we can see the time-series and the corresponding recurrence plots in the Grey and Spectrum layouts, of the three areas in Umbria (Norcia, Perugia and Terni). Perugia and Terni have a similar texture. Note that five recurrence plots in Figures 5 and 6, unless Pavia, are showing a boundary green band corresponding to a peak of healthy vegetation in 2014. Therefore, we can tell that recurrence plots based on the time-series of VHI could be interesting for the analysis of local environments.

\section{Comparing to NVDI}

From the same web service that provides the $\mathrm{VH}$ index data, we can also obtain NDVI data. Applying the same software used for the previous proposed discussion on $\mathrm{VH}$, we obtain the time-series of Figure 6 . Here we have selected just three areas, for the sake of simplicity (Trieste, Perugia and Pavia). In the Figure on the right, we can see one of the maps of No Noise NDVI maps from NOAA STAR. Note the evident seasonal behaviour of the time-series. It is also evident that, in the five years we considered, the average NDVI increases. Moreover, we can see that Perugia has, in average, the highest greenness parameter. In fact, the maps from NOAA demonstrate that Umbria is "the green heart of Italy", a widely used phrase, taken from a poem by Giosuè Carducci. In the lower part of the Figure, we can see the corresponding recurrence plots, with the typical image of an oscillating phenomenon. Note that Pavia has the widest oscillations, as remarked by the spanned colour tones. If we compare these recurrence plots to the ones given for VHI, we note that the periods of stressed environmental conditions are more clearly outlined by VHI recurrence plots.

\section{Conclusion}


In this paper, which adds a further contribution to the discussion proposed by one of us in Philica, article n.534, we proposed the use of recurrence plots for visualizing the behaviour of the NOAA STAR VH index. It is evident, comparing the plots of different areas, the role of local environments on the behaviour of VH Index. Further studies on this subject are in progress, to collect data from other parts of Italy and to compare VHI behaviours.

\section{References}

1. J.P. Eckmann, S. Oliffson Kamphorst, D. Ruelle (1987). Recurrence Plots of Dynamical Systems. Europhysics Letters 5 (9): 973-977. DOI: 10.1209/0295-5075/4/9/004

2. K. Fraedrich (1988). El Niño/Southern Oscillation Predictability. Monthly Weather Review 116(5):1001-1012. DOI: $10.1175 / 1520$ 0493(1988)116\%3C1001:enop\%3E2.0.co;2

3. N. Marwan (2003). Encounters with neighbours: current developments of concepts based on recurrence plots and their applications. Norbert Marwan.

4. A.C. Sparavigna (2014). Recurrence Plots from Altimetry Data of Some Lakes in Africa. International Journal of Sciences 3(07):19-27. DOI: 10.18483/ijSci.534

5. A.C. Sparavigna (2014). Carbon Dioxide Concentration and Emissions in Atmosphere: Trends and Recurrence Plots. International Journal of Sciences 3(10):8-15. DOI: 10.18483/ijSci.582

6. A.C. Sparavigna (2013). A Study of Moving Sand Dunes by Means of Satellite Images, International Journal of Sciences 2(08):33-42. DOI: $10.18483 / \mathrm{ijSci} .229$

7. A.C. Sparavigna 2013). The GNU Image Manipulation Program Applied to Study the Sand Dunes, International Journal of Sciences 2(09):1-8. DOI: 10.18483/ijSci.289

8. N. Marwan, J. Kurths, S. Foerster (2015). Analysing Spatially Extended High-Dimensional Dynamics by Recurrence Plots. Physics Letters A 379, 894-900. DOI: 10.1016/j.physleta.2015.01.013

9. S.C. Li , Z.Q. Zhao, F.Y. Liu (2008). Identifying Spatial Pattern of NDVI Series Dynamics Using Recurrence Quantification Analysis, A case study in the region around
Beijing, China. The European Physical Journal Special Topics 164(1):127-139. DOI: 10.1140/epjst/e2008-00839-y

10. D. M. Gates (1980). Biophysical Ecology, Springer-Verlag, New York. DOI: 10.1007/978-3-642-87810-7_1

11. R. D. Jackson, A. R. Huete (1991). Interpreting Vegetation Indices. Preventive Veterinary Medicine 11(3): 185-200. DOI: 10.1016/s0167-5877(05)80004-2

12. Jian Peng, Zhenhuan Liu, Yinghui Liu, Jiansheng Wu, Yinan Han (2012). Trend Analysis of Vegetation Dynamics in Qinghai-Tibet Plateau using Hurst Exponent. Ecological Indicators 14:28-39. DOI: 10.1016/j.ecolind.2011.08.011

13. Shuangshuang Li, Junping Yan, Xinyan Liu, Jia Wan (2013) Response of Vegetation Restoration to Climate Change and Human Activities in Shaanxi-Gansu-Ningxia Region, Journal of Geographical Sciences 23(1):98-112. DOI: 10.1007/s11442-013-0996-8

14. G. Katul, C.-T. Lai, K. Schäfer, B. Vidakovic, J. Albertson, D. Ellsworth, R. Oren (2001). Multiscale Analysis of Vegetation Surface Fluxes: from Seconds to Years Advances in Water Resources 24(9-10):1119-1132. DOI: 10.1016/s0309-1708(01)00029-x

15. Jihao Yin, Chao Gao ; Xiuping Jia (2012). Using Hurst and Lyapunov Exponent For Hyperspectral Image Feature Extraction. Geoscience and Remote Sensing Letters IEEE 9(4):705-709. DOI: 10.1109/LGRS.2011.2179005

16. J. C. Valdiviezo-N, R. Castro, G. Cristóbal, A. Carbone (2014). Hurst Exponent for Fractal Characterization of LANDSAT Images, Proc. SPIE 9221, Remote Sensing and Modeling of Ecosystems for Sustainability XI, 922103. DOI: $10.1117 / 12.2060281$

17. F. Kogan (2008). Monitoring Drought and its Impacts on Vegetation from Space. NIDIS Knowledge Assessment Workshop: Contributions of Satellite Remote Sensing to Drought Monitoring, February 6-7, 2008, David Skaggs Research Center, NOAA, Boulder, CO.

18. E. Kononov, Visual Recurrence Analysis, www.visualization-2002.org/

19. Wikipedia, retrieved on October 24, 2015 at https://en.wikipedia.org/wiki/Recurrence_plot

20. A. C. Sparavigna (2014). Recurrence Plots of Exchange Rates of Currencies, International Journal of Sciences 3(07):87-95. $\quad$ DOI: $\quad 10.18483 /$ ijSci.545 

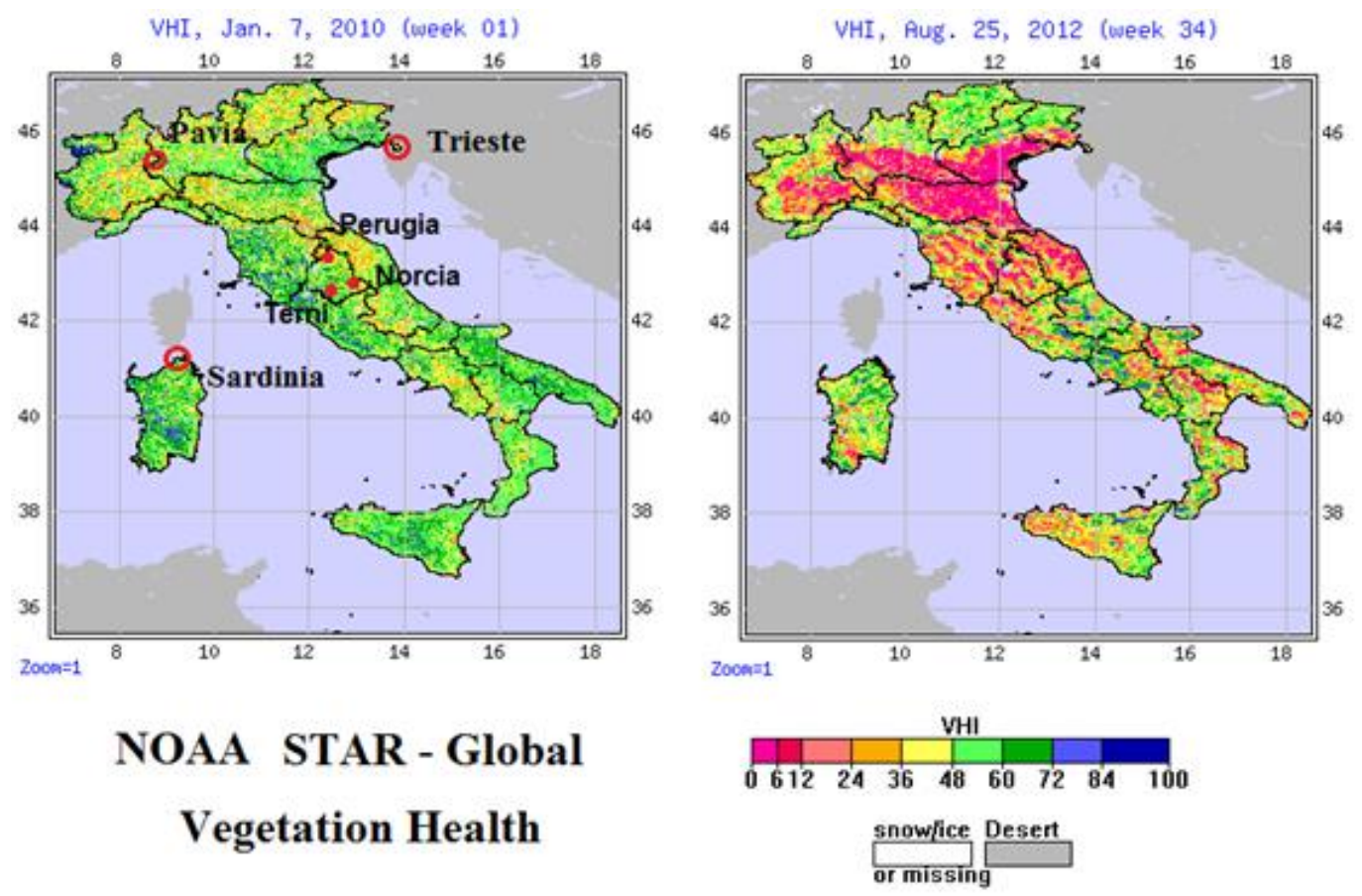

Figure 1: The figure shows two maps from NOAA STAR of Italy concerning Vegetation Health index. NOAA STAR is NOAA Center for Satellite Applications and Research. The behaviour of VH index for six Italian locations is considered. The three small areas encircled in the upper/left image (Pavia, Trieste, Sardinia) are supposed to have quite different behaviours, whereas the three locations in Umbria (Perugia, Norcia and Terni) are supposed to have the same behaviour.

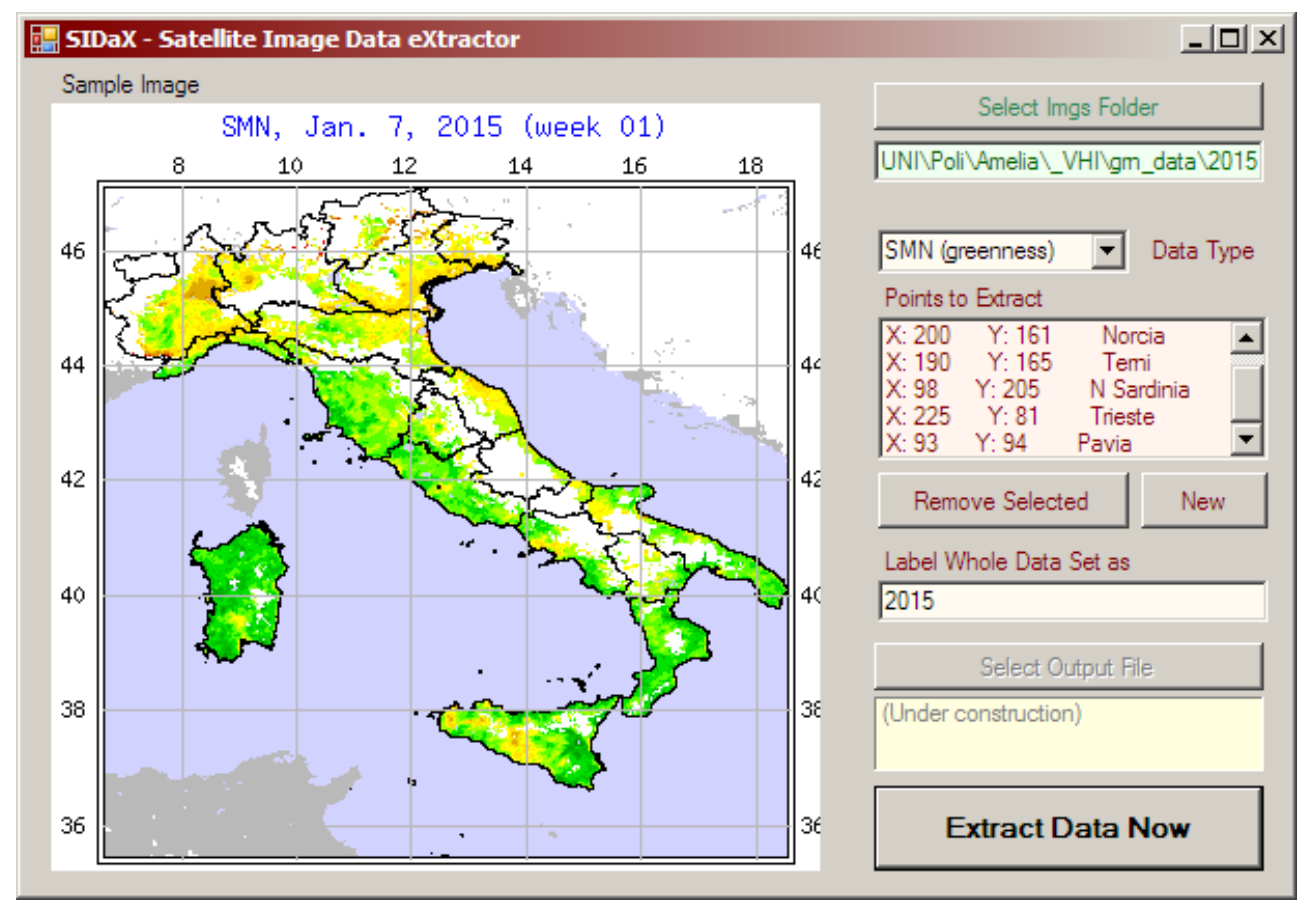

Figure 2: Screenshot of SIDaX - Satellite Image Data eXtractor, developed for the present work on vegetation indices. 


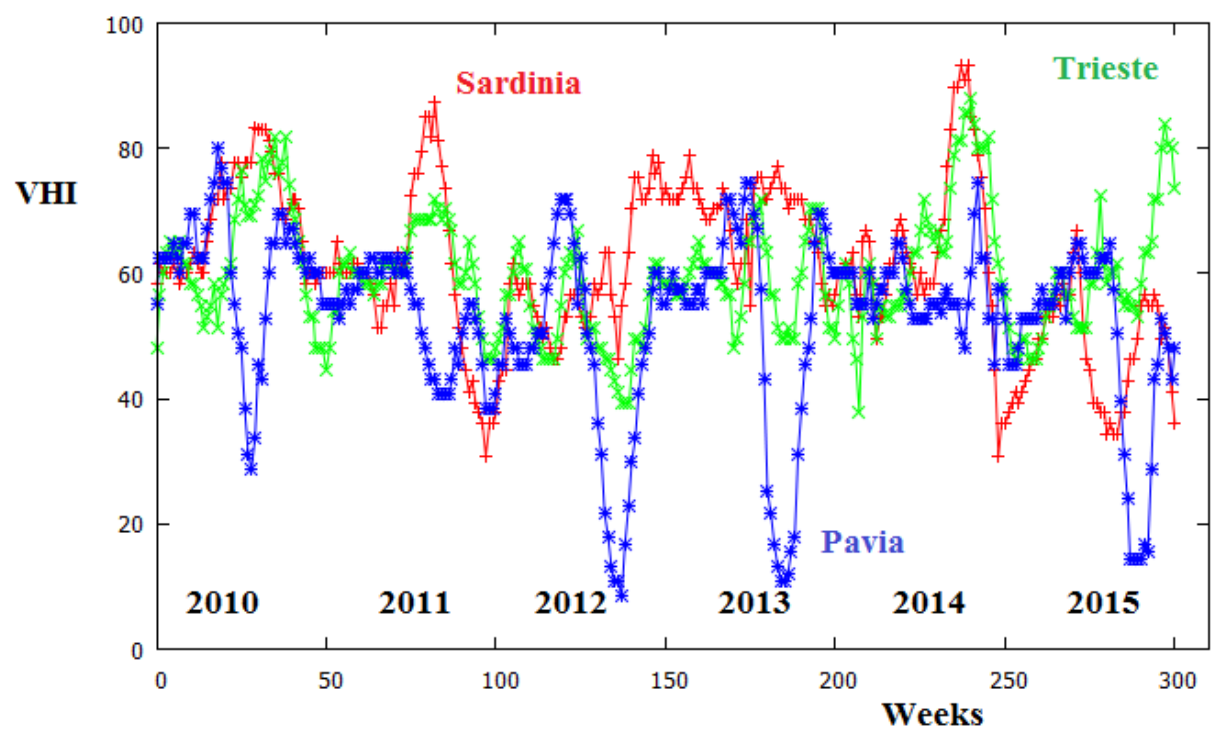

Figure 3: Time-series of the selected areas of Sardinia, Trieste and Pavia. Note the different behaviour of Pavia, which has peaks of stressed vegetation. Let us note also that high values correspond to healthy vegetation, whereas lower values represent a stressed situation.

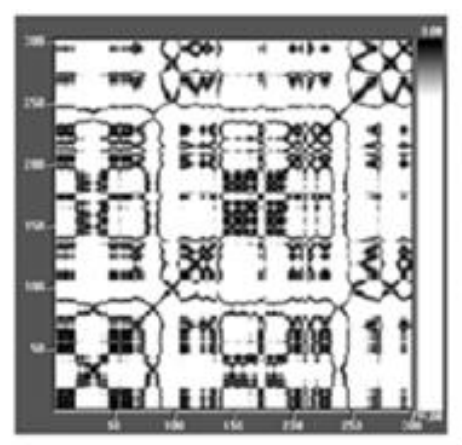

Sardinia

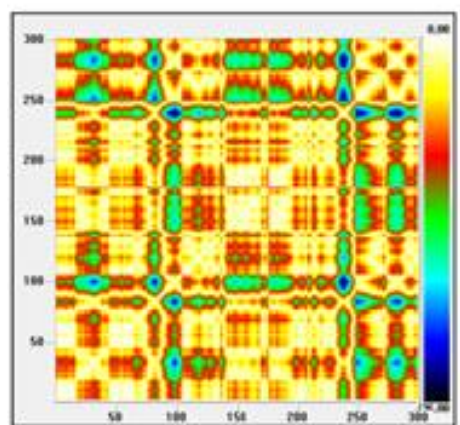

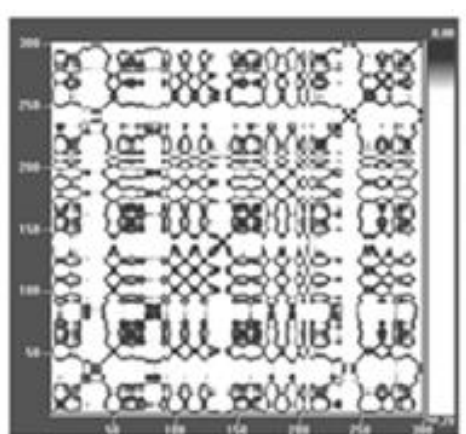

Trieste

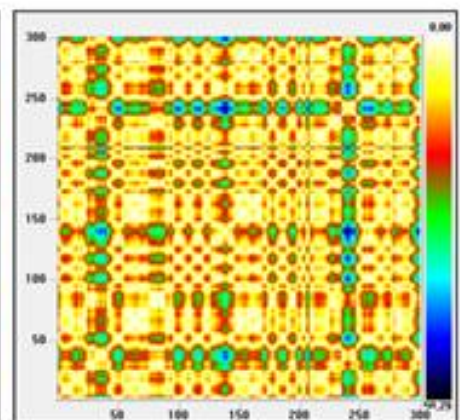

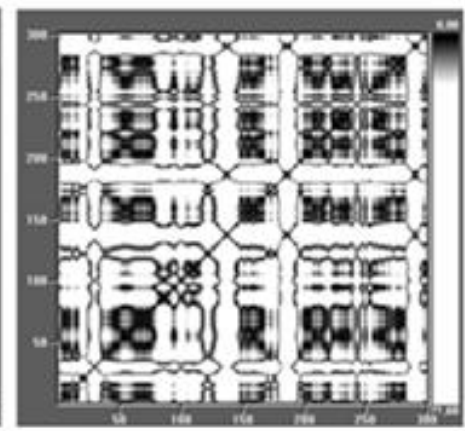

Pavia

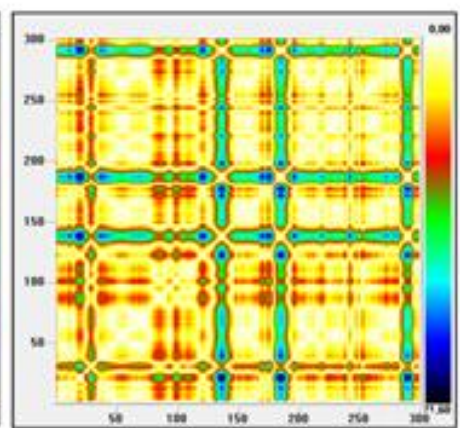

Figure 4: From time-series of VHI given in Fig.3, we can find the corresponding recurrence plots. In the upper plots, black or dark grey pixels correspond to high recurrence, whereas white pixels to low or very low recurrence. The plots look like a mixture of oscillatory and autoregressive processes. Note the strong stresses of vegetation, which are connected to those observed for the Pianura Padana as a whole. In the lower plots, a coloured layout is used, where white pixels correspond to high recurrence. 

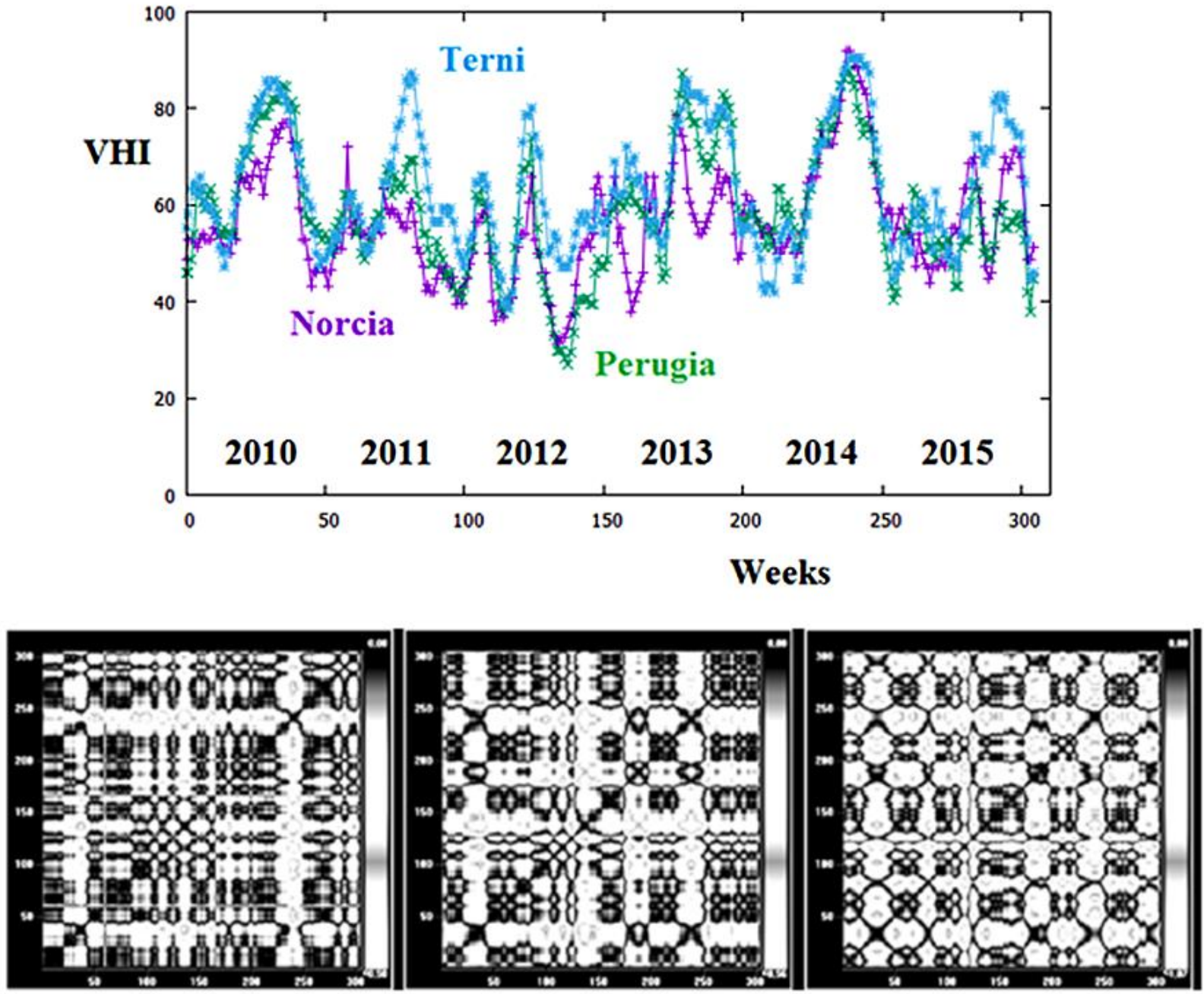

Norcia

Perugia

Terni
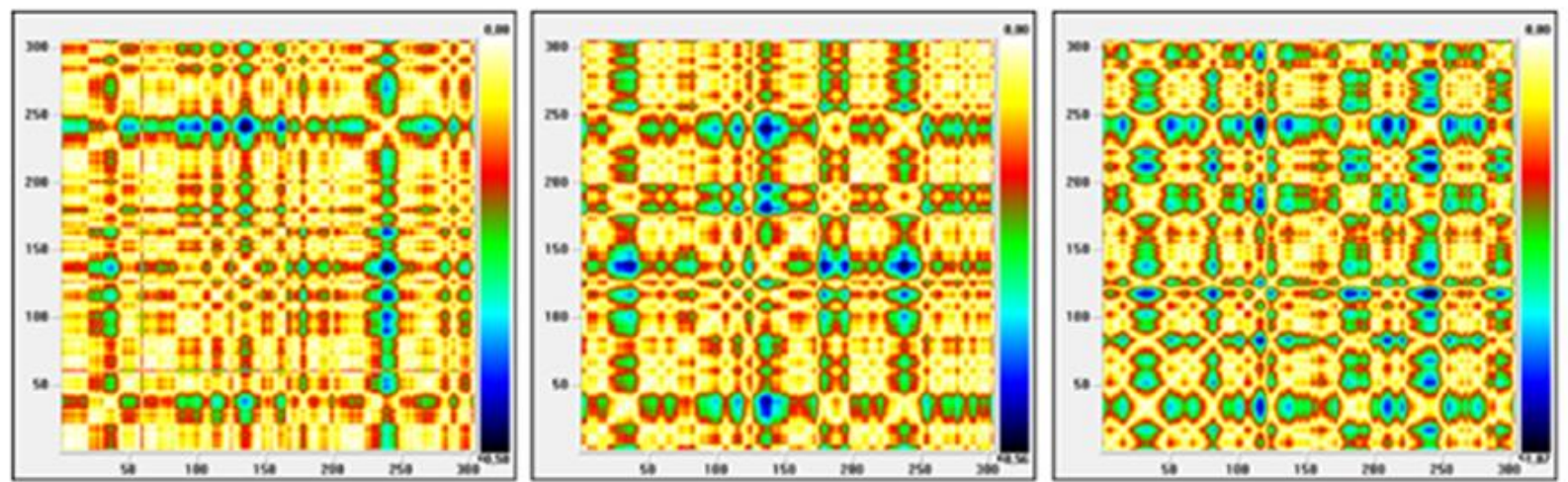

Figure 5: Time-series and corresponding recurrence plots of the selected areas in Umbria (Norcia, Perugia and Terni). In the upper plots, black or dark grey pixels correspond to high recurrence, whereas white pixels to low or very low recurrence. Again, we have that the plots look like a mixture of oscillatory and autoregressive processes. In the lower plots, a coloured layout is used, in which white pixels correspond to high recurrence. 


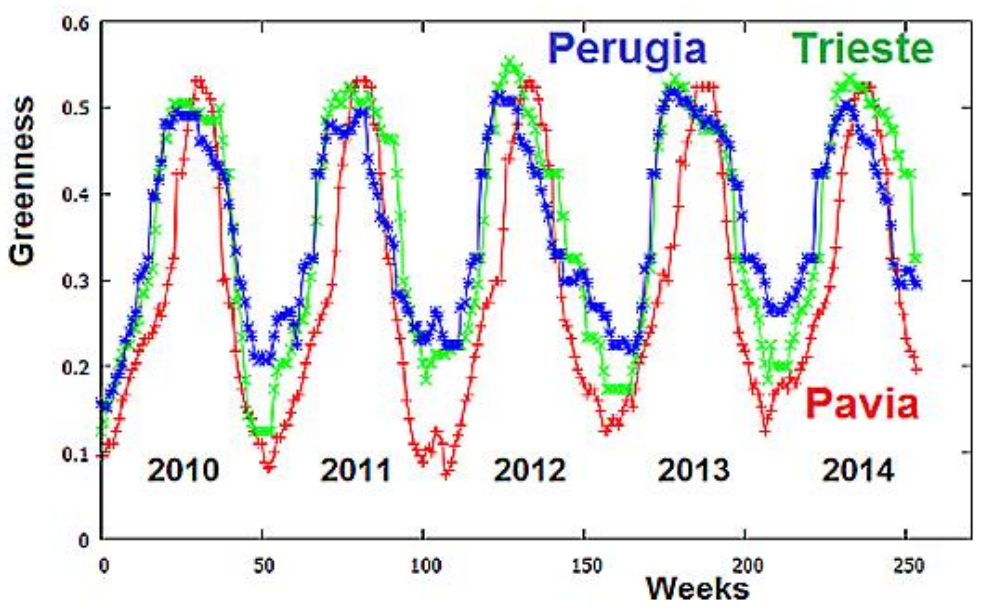

Italy - Greenness (No Noise NDVI)

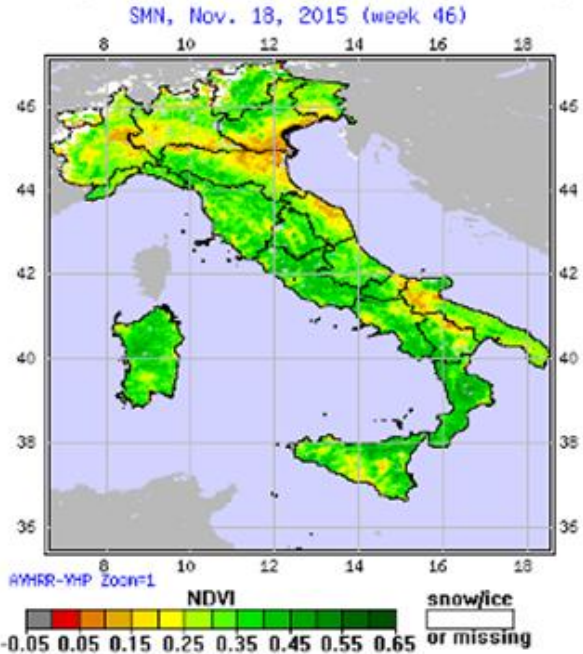

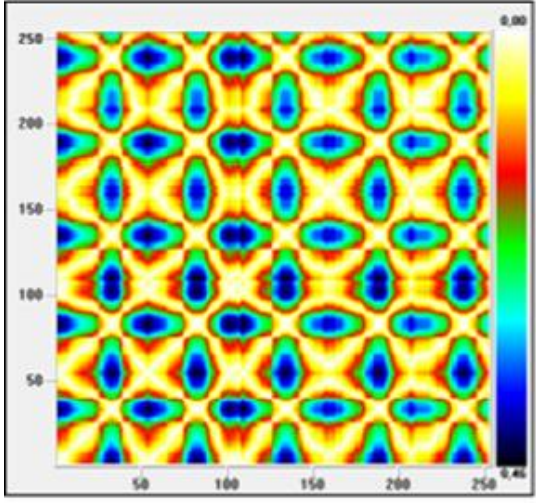

Pavia

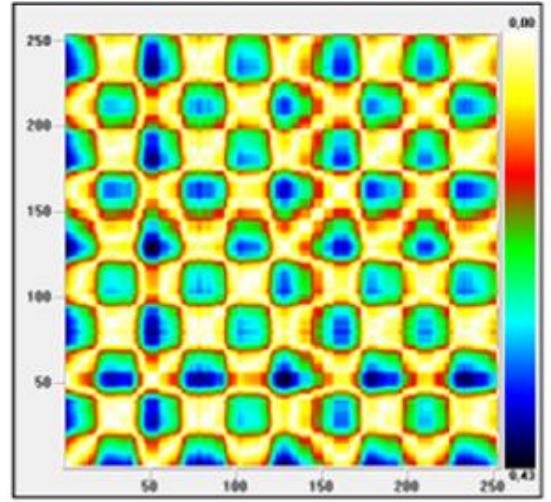

Trieste

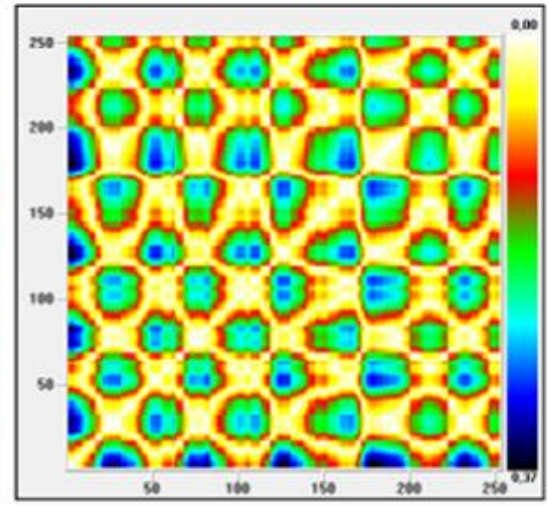

Perugia

Figure 6: Time-series of the selected areas of Trieste, Perugia and Pavia, for NDVI maps from NOAA STAR. Note the clear evident seasonal behaviour of the peaks. It is also evident that, in the five years we considered, the NDVI average increases. Moreover, we can also see that Perugia has, in average, the highest greenness. In fact, the maps from NOAA demonstrate that Umbria is "the green heart of Italy", a widely used phrase, taken from a poem by Giosuè Carducci. In the lower part of the Figure, we can see the corresponding recurrence plots, with the typical image of an oscillating phenomenon. 